\title{
Triamcinolone Acetonide in the Treatment of Perennial Allergic Rhinitis: A post hoc Analysis of Quality of Life during a Phase III Study
}

\author{
Alexander V. Karaulov ${ }^{\mathrm{a}}$ Natalia Nenasheva ${ }^{\mathrm{b}}$ Yury Smolkin ${ }^{\mathrm{c}}$ \\ Aleksandr Maslakov ${ }^{d}$ Luiz Lucio $^{\text {e }}$ \\ aDepartment of Clinical Immunology and Allergology, I.M. Sechenov First Moscow State Medical University, \\ Moscow, Russia; bepartment of Immunology and Allergology, Russian Medical Academy for Continued \\ Professional Education, Moscow, Russia; 'Department of Clinical Immunology and Allergology, Research and \\ Clinical Center for Allergy and Clinical Immunology, Moscow, Russia; ${ }^{\mathrm{d}}$ Medical Department, Sanofi Consumer \\ Healthcare, Moscow, Russia; 'Medical Department, Sanofi Consumer Healthcare, São Paulo, Brazil
}

\section{Keywords}

Allergic rhinitis · Triamcinolone acetonide · Quality of life · Allergic conjunctivitis · Perennial allergic rhinitis

\begin{abstract}
Introduction: Allergic rhinitis (AR) is a disease that affects $\leq 24 \%$ of people in Russia, significantly impairing quality of life (QoL). Intranasal corticosteroids, such as triamcinolone acetonide (TAA), are considered effective drugs for treatment. A post hoc analysis of data (phase III NASANIF trial) examined weekly QoL changes in patients receiving TAA for the treatment of perennial AR (PAR). Methods: NASANIF (NCT03317015) was a double-blind, parallel group, multicenter, prospective, noninferiority, phase III clinical trial. Patients with PAR were randomized (1:1) to receive TAA or fluticasone propionate (FP) for 4 weeks. Here, a post hoc analysis measures QoL using a shortened Rhinoconjunctivitis Quality of Life Questionnaire (miniRQLQ). Differences in miniRQLQ score were evaluated using a mixed linear model and descriptive statistics. A subgroup analysis was performed in patients with a previous diagnosis of allergic conjunctivitis. $\boldsymbol{R e}$ sults: Of 260 patients eligible for randomization, 128 each completed treatment with TAA or FP. Overall and individual
\end{abstract}

domain scores progressively improved and were significantly different versus baseline at week 4 in both treatment groups: LS mean difference TAA: $-30.92(95 \% \mathrm{Cl}[-33.01$ to $-28.83]), p<0.001$, and FP: -31.13 (-33.23 to -29.04$), p<$ 0.001 . In both arms of the subgroup, there was a significant reduction in eye symptoms. There was no significant difference between the TAA and FP treatment groups in any analyses. Conclusions: TAA is effective in improving overall and individual domains of QoL in patients with PAR, over 4 weeks. Patients with a previous diagnosis of allergic conjunctivitis experienced significant improvements in QoL related to the resolution of these symptoms.

(c) 2021 The Author(s).

Published by S. Karger AG, Basel

\section{Introduction}

Allergic rhinitis (AR) is a multifactorial disease that can be classified as either seasonal or perennial (PAR). In accordance with the Allergic Rhinitis in the Impact of Asthma (ARIA) guidelines, AR can also be classified based on the duration of the disease, intermittent or per-

Edited by: H.-U. Simon, Bern.

Correspondence to:

Alexander V. Karaulov, drkaraulov@mail.ru

karger@karger.com www.karger.com/iaa

Karger $\stackrel{\text { ' }}{=}$

BOPEN ACCESS
(C) 2021 The Author(s)

Published by S. Karger AG, Basel

This is an Open Access article licensed under the Creative Commons Attribution-NonCommercial-4.0 International License (CC BY-NC) (http://www.karger.com/Services/OpenAccessLicense), applicable to the online version of the article only. Usage and distribution for commercial purposes requires written permission. 
sistent, or based on severity, that is, mild, moderate, or severe [1]. AR is a widespread health problem that affects up to $24 \%$ of the population in Russia [2].

Nasal symptoms of AR include rhinorrhea, sneezing, itching, and obstruction, which can impair quality of life (QoL). While nasal symptoms are those that are often described as the most troublesome by patients, eye symptoms are also an important and overlooked effect of AR as they can greatly impact QoL [3]. Furthermore, studies have shown that AR significantly influences many other areas of life, such as work productivity $[4,5]$, highlighting the importance of effective intervention in countries (such as Russia) where it is prevalent. Intranasal corticosteroids are considered to be the most effective drugs for treating AR symptoms, as they provide precise treatment by reducing inflammation in the nasal mucosa [6]. Delivering high drug concentrations directly to the area of allergic inflammation inhibits reactivity to allergens and effectively improves symptoms [7].

Triamcinolone acetonide (TAA) is a synthetic fluorinated corticosteroid approved for the treatment of AR in adults and pediatric patients [8]. In the phase III NASANIF trial, which was conducted in Russia, TAA was shown to have a good safety profile and noninferior efficacy to FP in adults with persistent PAR [9]. We undertook a post hoc analysis of the NASANIF trial to examine and quantify weekly changes in QoL during the course of treatment with TAA or FP.

\section{Methods}

\section{Study Design}

The NASANIF trial was a randomized (1:1), double-blind, parallel group, multicenter, prospective, noninferiority, phase III clinical trial (NCT03317015) conducted between November 30, 2016, and July 10, 2017, in 12 study centers in Russia. The study design has been published previously [9]. In brief, 260 patients with previously diagnosed persistent PAR were randomized (1:1) to receive either intranasal TAA ( $220 \mu \mathrm{g} / \mathrm{day}$, administered as $2 \times$ $55 \mu \mathrm{g}$ sprays per nostril) or intranasal FP ( $200 \mu \mathrm{g} /$ day, administered as $2 \times 50 \mu \mathrm{g}$ sprays per nostril) for 4 weeks. In the original study, QoL was assessed at baseline and week 4 using a patientadministered short version of the Rhinoconjunctivitis Quality of Life Questionnaire (miniRQLQ), which contains 14 items in 5 domains: activity limitations, practical problems, nasal symptoms, eye symptoms, and other symptoms. All items on the miniRQLQ are scored using a 7-point response scale (online suppl. Table S1; see www.karger.com/doi/10.1159/000518753 for all online suppl. material) [10]. In this post hoc analysis, QoL scores were calculated, both overall and for individual domains, at baseline (analysis day 1), week 1 (analysis days 8-10), week 2 (analysis days 15-17), week 3 (analysis days 22-24), and week 4 (analysis days 29-31) [10].

Quality of Life Improvement by

Triamcinolone Acetonide in PAR

\section{Study Populations}

The intent-to-treat (ITT) population included all randomized patients who met the inclusion criteria, none of the exclusion criteria, and took at least 1 dose of study medication. The per-protocol population included all patients from the ITT population who complied with the protocol and completed the study with no protocol violations and was used for the main analyses.

\section{Subgroup Analysis}

A subgroup analysis was performed of patients in the per-protocol population who had eye symptoms at baseline. This subgroup of eye symptoms was defined by a previous diagnosis of concomitant allergic conjunctivitis, as informed by the patients. These patients were identified from data gathered during screening.

\section{Statistical Methods}

Mean overall and individual domain scores were calculated weekly for each treatment, both for the overall study population and the subgroup of patients with comorbid allergic conjunctivitis. Scores for individual components of the "eye symptoms" domain were also analyzed.

Changes from baseline in miniRQLQ scores were calculated and analyzed using a linear mixed model. To calculate and compare least-squares (LS) means, a mixed model with categorical time (weeks of treatment), random intercept, treatment, and time/ treatment interaction terms was estimated. No simplification or building strategy was applied to these models. For comparisons between visits, LS mean differences were reported together with 95\% confidence intervals (CIs). Comparisons between treatments were calculated, and significance was reported using 2 -sided $p$ values from the mixed model.

The main analyses were performed on the per-protocol population, and sensitivity analyses were performed on the ITT population. All statistical analyses were performed using SAS ${ }^{\circledR}$ Version 9.4 (Cary, NC, USA). In general, substitution of missing data was not done; missing data were not imputed but instead were handled as "missing" in the statistical evaluation.

\section{Results}

Two hundred and sixty patients were randomized (TAA, $n=129$; FP, $n=131$ ), and 256 completed the 4 -week trial ( $n=128$ per arm). Patient population, baseline demographics, and clinical characteristics were previously reported [9].

\section{Overall miniRQLQ Score}

The mean (standard deviation) overall miniRQLQ scores at baseline were 38.14 (13.45) for TAA and 38.56 (13.63) for FP. Mean overall scores were significantly reduced ( $p<0.001$ ), versus baseline, in each week of treatment in both treatment groups (Table 1; Fig. 1). At week 4 , the LS mean $(95 \% \mathrm{CI})$ change from baseline was -30.92 $(-33.01$ to -28.83$)$ in the TAA $\operatorname{arm}(p<0.001)$ and -31.13

Int Arch Allergy Immunol 2022;183:160-167 
Table 1. Change from baseline in least-squares mean overall score and scores for individual domains of the miniRQLQ

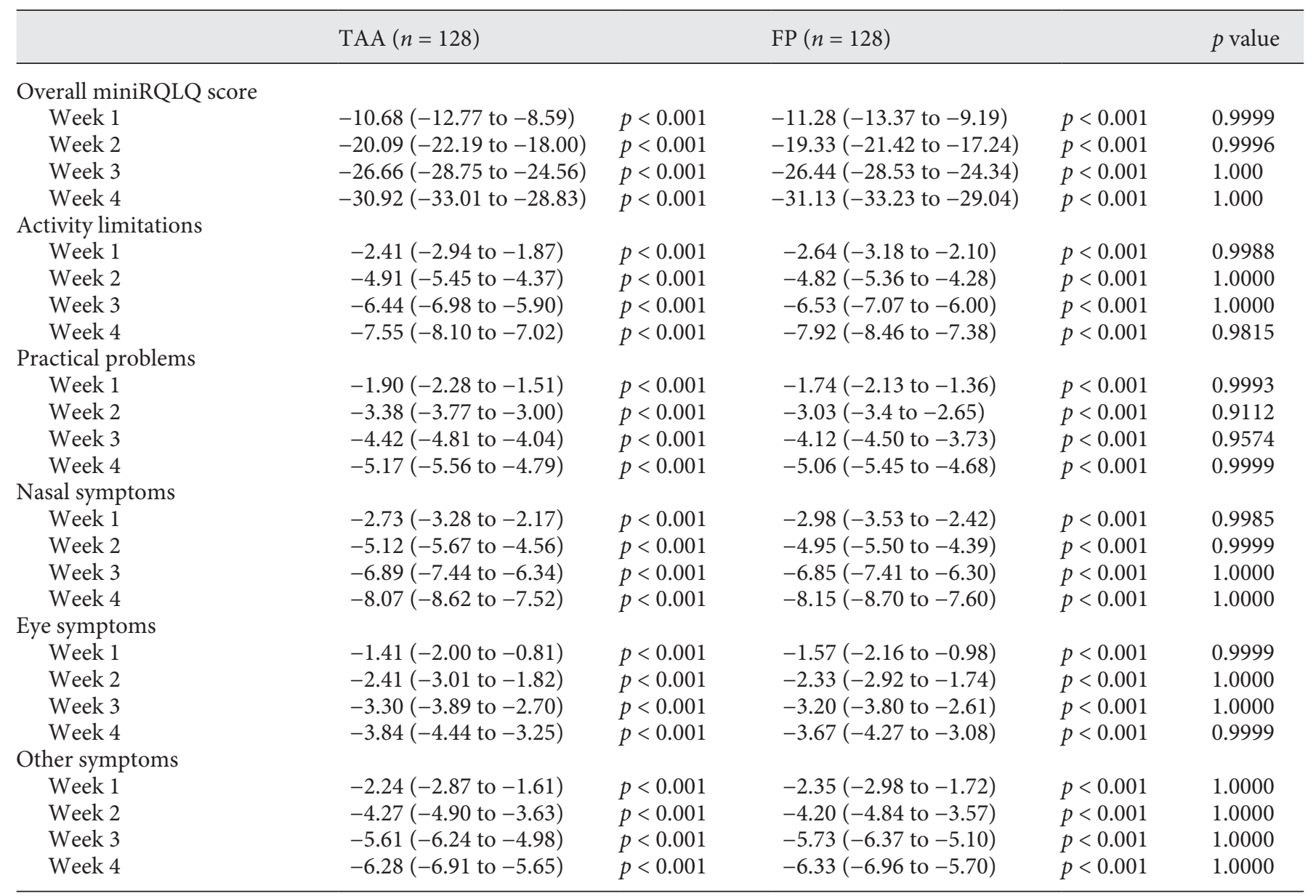

Data in brackets show 95\% confidence intervals. FP, fluticasone propionate; miniRQLQ, mini-Rhinoconjunctivitis Quality of Life Questionnaire; TAA, triamcinolone acetonide.

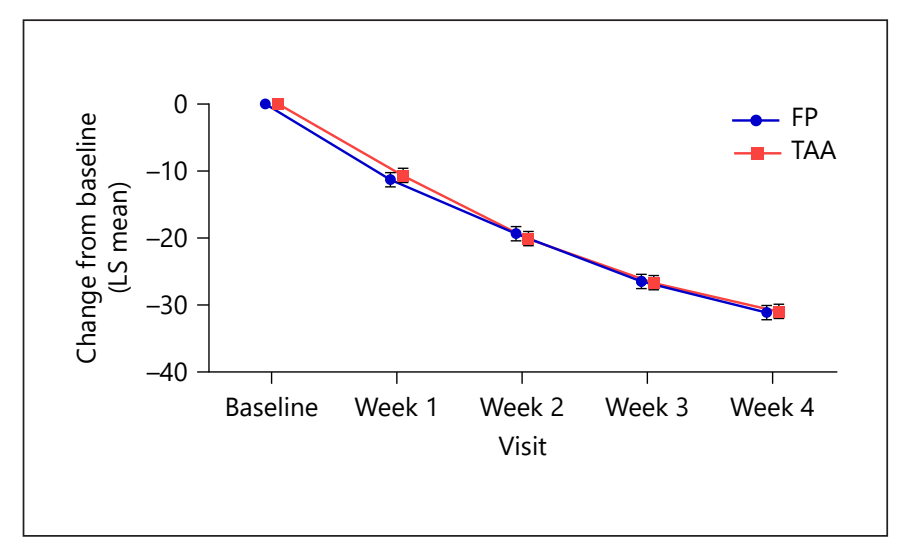

Fig. 1. LS mean change in overall miniRQLQ score versus baseline. Error bars show the standard error of the mean. LS, least-squares; FP, fluticasone propionate; miniRQLQ, mini-Rhinoconjunctivitis Quality of Life Questionnaire; TAA, triamcinolone acetonide.
$(-33.23$ to -29.04$)$ in the FP arm $(p<0.001)$. There were no significant differences between treatment groups.

\section{Individual miniRQLQ Domains}

Scores in each of the 5 domains (activity limitations, practical problems, nasal symptoms, eye symptoms, and other symptoms) significantly reduced $(p<0.001)$ from baseline in each week of treatment with TAA or FP (Table 1; Fig. 2). The greatest change from baseline in the TAA group was seen in nasal symptoms, with an LS mean (95\% CI) reduction of -8.07 ( -8.62 to -7.52$)$ at week 4 $(p<0.001)$ (Table 1). Similar reductions were observed in the FP treatment group in each analysis, with no significant differences between groups.

Within the eye symptoms domain, scores for individual items (symptoms) also significantly decreased $(p<$ 


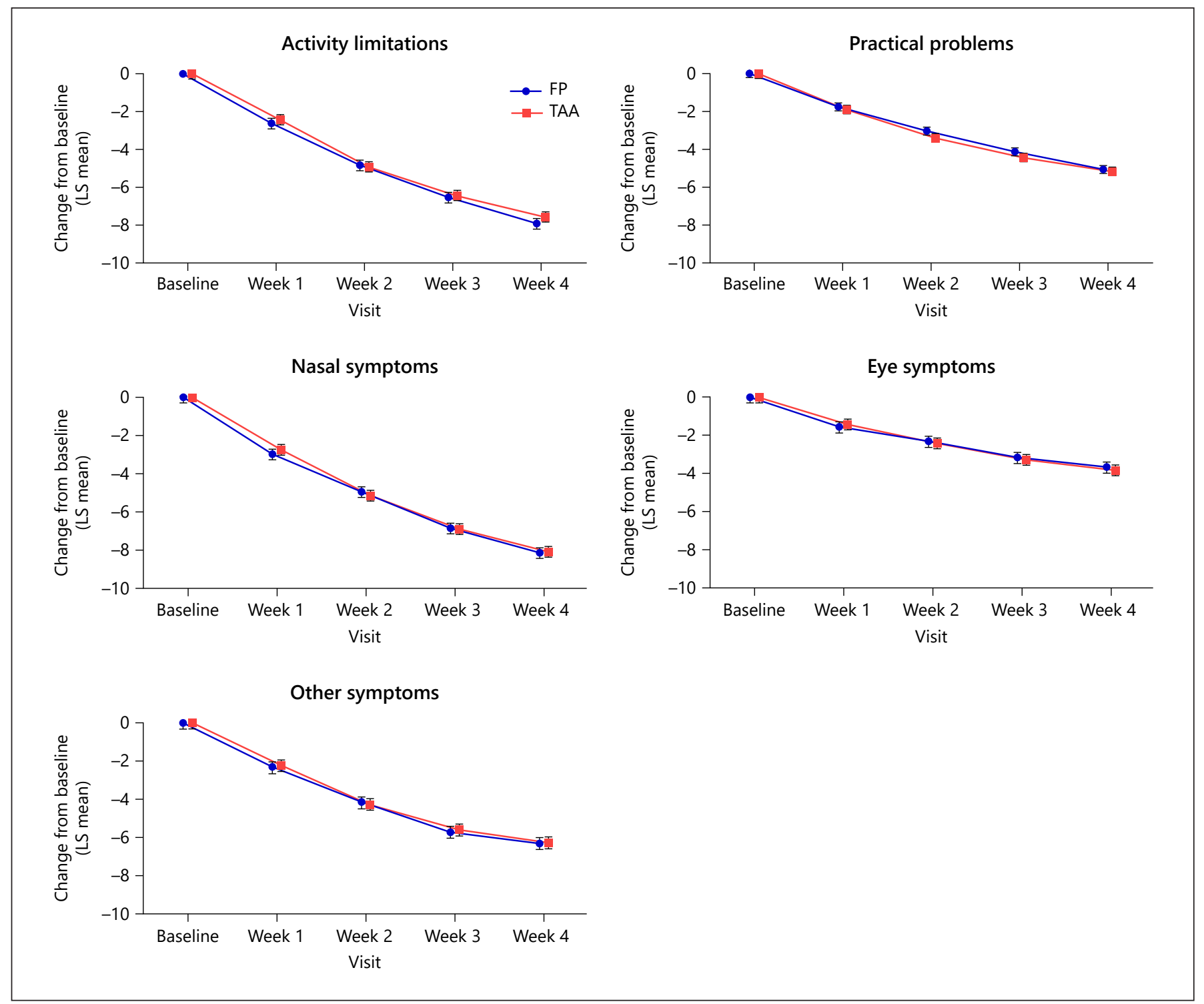

Fig. 2. LS mean change in miniRQLQ scores, versus baseline, for each of the 5 individual domains. Error bars show the standard error of the mean. LS, least-squares; FP, fluticasone propionate; miniRQLQ, mini-Rhinoconjunctivitis Quality of Life Questionnaire; TAA, triamcinolone acetonide.

0.001) per week of treatment (Table 2). The greatest change from baseline in the TAA group was in itchy eyes, with an LS mean $(95 \% \mathrm{CI})$ reduction of $-1.45(-1.68$ to -1.23 ) at week 4 . Similar reductions were observed in the FP treatment group in each analysis, with no significant differences between groups.

\section{Subgroup Analysis}

The subgroup analysis included 37 patients from the TAA arm and 43 patients from the FP arm. After
4 weeks of treatment, overall miniRQLQ scores had greatly decreased versus baseline (Fig. 3), with LS mean $(95 \% \mathrm{CI})$ reductions of $-34.84(-38.85$ to -30.83$)$ and $-33.02(-36.74$ to -29.30$)$ for TAA and FP, respectively (online suppl. Table 2); no significant difference was observed between the TAA and FP treatment groups. In the subgroup, successive weekly reductions in scores were also seen in individual domains (online suppl. Table 2) and for individual components of the eye symptom domain (Fig. 4). Similar reduc- 


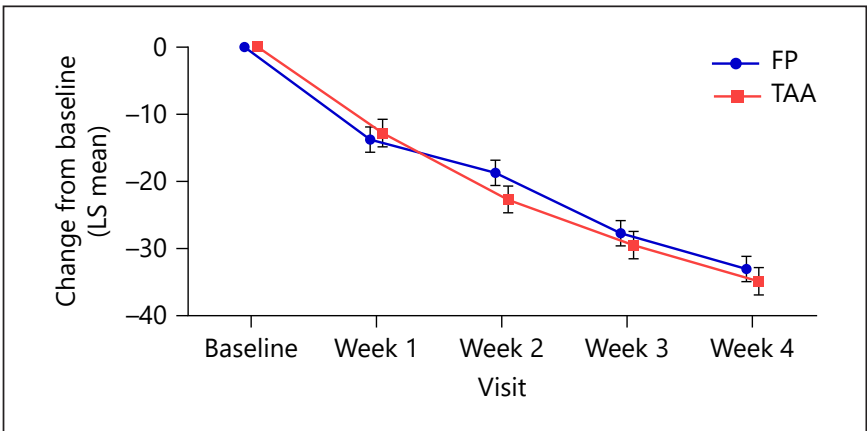

Fig. 3. LS mean change in overall miniRQLQ score versus baseline in patients with concomitant allergic conjunctivitis. Error bars show the standard error of the mean. LS, least-squares; FP, fluticasone propionate; miniRQLQ, mini-Rhinoconjunctivitis Quality of Life Questionnaire; TAA, triamcinolone acetonide.

tions were observed in the FP treatment group in each analysis, with no significant differences between groups.

\section{Discussion}

The intranasal administration of TAA or FP significantly improved miniRQLQ scores, both overall and in the 5 individual domains, over 4 weeks in patients with PAR in Russia. Other studies have demonstrated improvements in QoL in patients with seasonal or persistent AR who received TAA $[7,11,12]$. Our analysis has been broadened to study the effects of TAA in patients with PAR. The change in miniRQLQ score from baseline was statistically significant at each week of treatment, and there was no statistically significant difference between treatments. Considering the low proportion of patients who had a previous diagnosis of eye symptoms at baseline, we performed a subgroup analysis of these patients and found that the improvement of eye symptoms by TAA in these patients was far greater than that shown in the overall study population.

Intranasal corticosteroids are most commonly associated with improvements in nasal symptoms due to their localized effects in the nasal tissue [13]. These treatments act in the early and late phases of allergic reactions [14] and have been shown to be superior to oral corticosteroids and antihistamines, particularly in the treatment of nasal symptoms $[15,16]$. Previous trials have shown that TAA is effective in reducing these symptoms in patients with $\operatorname{AR}[9,17]$, and the results of our post hoc analysis
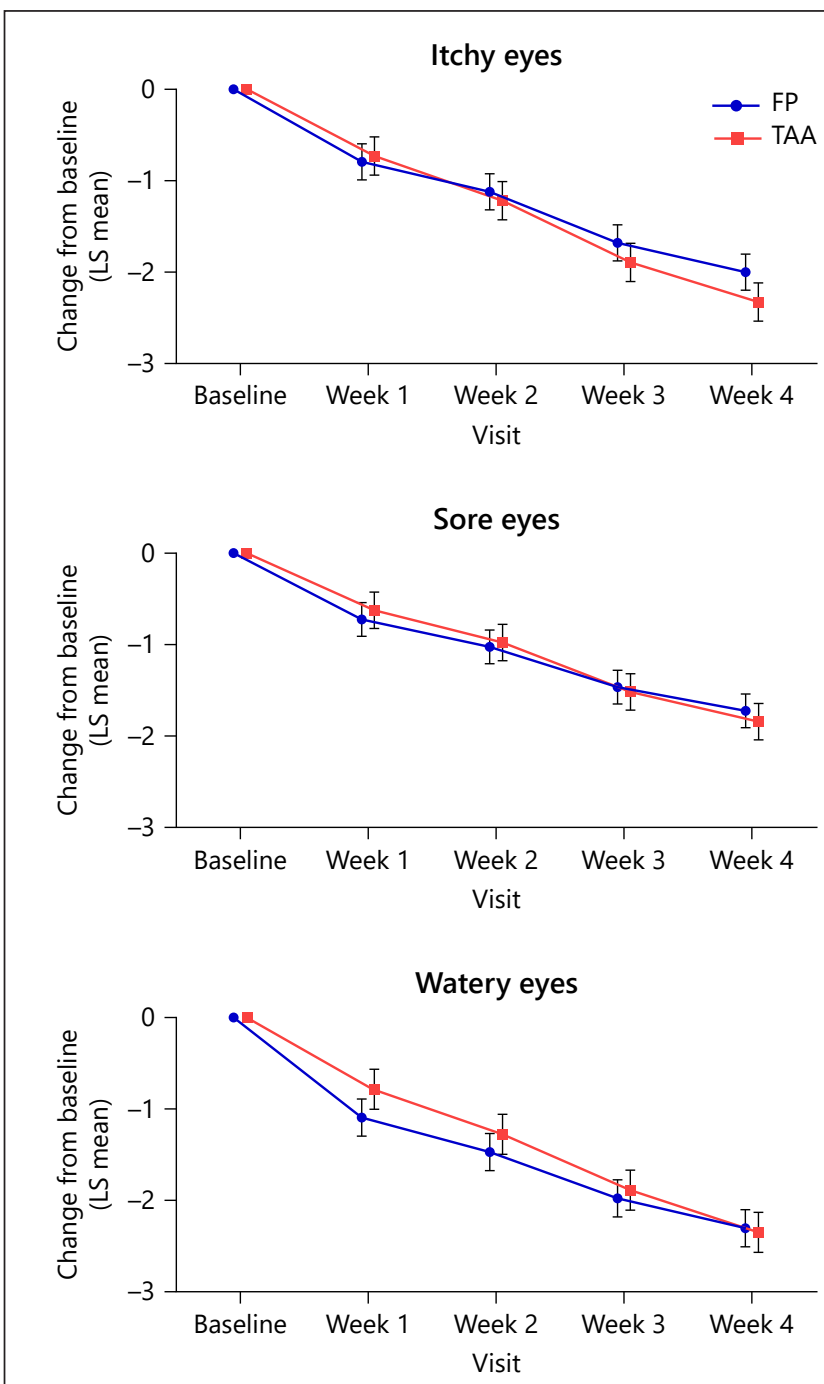

Fig. 4. LS mean change in miniRQLQ scores, versus baseline, for the eye symptoms domain in patients with concomitant allergic conjunctivitis. Error bars show the standard error of the mean. FP, fluticasone propionate; miniRQLQ, mini-Rhinoconjunctivitis Quality of Life Questionnaire; TAA, triamcinolone acetonide; LS means: least-squares means.

are consistent with these findings. Furthermore, we have shown that TAA provides effective and prolonged improvement of PAR symptoms in another post hoc analysis of the same trial (Karaulov et al. [18], submitted).

Nasal symptoms of AR can impair QoL including interfering with sleep, most likely due to nasal congestion, and AR-related sleep disturbance is a defining feature of severe AR that has direct effects on fatigue, mood, and daytime somnolence [19]. In an open-label primary care 
Table 2. Change from baseline in least-squares mean scores for individual items in the "eye symptoms" domain of the miniRQLQ

\begin{tabular}{cllll}
\hline & TAA $(n=128)$ & & FP $(n=128)$ & $p$ value \\
\hline Watery eyes & & & & \\
Week 1 & $-0.52(-0.74$ to -0.29$)$ & $p<0.001$ & $-0.60(-0.83$ to -0.37$)$ & $p<0.001$ \\
Week 2 & $-0.83(-1.06$ to -0.60$)$ & $p<0.001$ & $-0.87(-1.10$ to -0.64$)$ & $p<0.001$ \\
Week 3 & $-1.14(-1.37$ to -0.91$)$ & $p<0.001$ & $-1.20(-1.43$ to -0.97$)$ & $p<0.001$ \\
Week 4 & $-1.36(-1.59$ to -1.13$)$ & $p<0.001$ & $-1.34(-1.57$ to -1.12$)$ & $p<0.001$ \\
Itchy eyes & & & & 0.9995 \\
Week 1 & $-0.49(-0.72$ to -0.27$)$ & $p<0.001$ & $-0.54(-0.77$ to -0.31$)$ & $p<0.001$ \\
Week 2 & $-0.92(-1.15$ to -0.70$)$ & $p<0.001$ & $-0.85(-1.08$ to -0.62$)$ & $p<0.001$ \\
Week 3 & $-1.25(-1.48$ to 1.02$)$ & $p<0.001$ & $-1.19(-1.41$ to -0.96$)$ & $p<0.001$ \\
Week 4 & $-1.45(-1.68$ to -1.23$)$ & $p<0.001$ & $-1.34(-1.57$ to -1.12$)$ & $p<0.001$ \\
Sore eyes & & & 0.9999 \\
Week 1 & $-0.40(-0.60$ to -0.19$)$ & $p<0.001$ & $-0.43(-0.64$ to -0.22$)$ & 0.9999 \\
Week 2 & $-0.66(-0.90$ to -0.46$)$ & $p<0.001$ & $-0.61(-0.82$ to -0.40$)$ & $p<0.001$ \\
Week 3 & $-0.91(-1.11$ to -0.70$)$ & $p<0.001$ & $-0.81(-1.02$ to 0.61$)$ & $p<0.001$ \\
Week 4 & $-1.03(-1.24$ to -0.83$)$ & $p<0.001$ & $-0.98(-1.19$ to -0.78$)$ & $p<0.001$ \\
\end{tabular}

Data in brackets show 95\% confidence intervals. FP, fluticasone propionate; miniRQLQ, mini-Rhinoconjunctivitis Quality of Life Questionnaire; TAA, triamcinolone acetonide.

study, TAA was found to improve both sleep quality and nocturnal rhinitis-related QoL [19].

Additionally, intranasal corticosteroids also have a significant impact on nonnasal symptoms of AR. Eye symptoms, such as itching, lacrimation, and eyelid edema, are troublesome to patients and can significantly impair QoL [3]. In previous studies, TAA has been shown to improve eye symptoms, particularly lacrimation $[14,15]$. In addition, a 2020 meta-analysis by Bielory et al. [17] found a significant reduction in the occurrence of eye symptoms, especially watery eyes, in patients treated with TAA. In our analysis, among the 128 patients who completed 4 -week treatment with TAA, only 37 had a previous diagnosis of eye symptoms. A subgroup analysis of these patients was therefore performed to minimize potential bias introduced by the inclusion of patients with no eye symptoms at baseline and to better characterize the effects of TAA on these symptoms. This analysis showed that changes from baseline in overall miniRQLQ scores were greater, at each week of the study, in the subgroup compared with the whole study population. The main post hoc analysis showed change from baseline data for the eye symptom domain that is suggestive of a dramatic reduction in eye symptoms at week 4 , and the subgroup analysis reinforces this. In patients with AR, intense eye symptoms are mostly associated with seasonal AR [17], but our findings suggest that eye symptoms can impact QoL of at least some patients with PAR. The improvements in eye symptom scores during our study suggest that TAA has the potential to improve QoL in patients for whom eye symptoms are troublesome.

TAA has similar efficacy and safety to other intranasal corticosteroids in the treatment of AR $[11,20]$; however, the bioavailability of some intranasal corticosteroids including TAA is much higher (23-46\%) than others such as FP and mometasone $(<1 \%)[21,22]$. The primary concern of high bioavailability is related to potential adverse events; however, bioavailability of an intranasal corticosteroid does not predict the incidence of adverse events nor the efficacy. Many different properties of each intranasal corticosteroid have a direct or indirect correlation that will determine their safety and efficacy, such as volume of distribution, tissue deposition, receptor binding targeting, tissue retention, lipophilicity, glucocorticoid potency, systemic potency, and elimination half-life [14, $23,24]$. Comparative studies that have evaluated TAA and FP demonstrated a similar safety profile of these intranasal corticosteroids despite the difference in bioavailability $[9,11,12]$; therefore, TAA can be considered a well-tolerated choice.

TAA is often a preferred choice by patients because of its perceived sensory attributes (e.g., feel of spray in nose and throat) [20]. In addition, compared with other intranasal steroids, TAA is associated with less odor, less aftertaste, and greater overall patient liking [25]. Patient satisfaction with efficacy and sensorial attributes of treatment 
is likely to influence adherence and, consequently, the real-world effectiveness of treatment [25]. During the initial phase III NASANIF trial, patient and physician satisfaction was included as a secondary endpoint and measured on a 5-point scale. Most patients and physicians were satisfied with TAA treatment after 4 weeks, a finding that was consistent with the improvements of QoL seen across domains; a majority of patients showed substantial improvement after 4 weeks in both treatment groups; $85.5 \%$ of patients ( $83.6 \%$ for TAA and $87.5 \%$ for FP). Regarding physicians, $87.9 \%$ of reported substantial improvement in both treatment groups: $84.4 \%$ with TAA and $91.4 \%$ for FP (online suppl. Table 3) [9].

We acknowledge the limitations of our study. We did not compare improvements in QoL scores for subgroups of interest defined by age, sex, or ethnicity. Our study may also be limited by its short duration in the context of a year-round disease, and more data are needed to see whether the effects of TAA on QoL are sustained over a longer period.

In conclusion, improvement in QoL should be given due consideration in the treatment of $\mathrm{AR}$, due to its high prevalence. This post hoc analysis demonstrates that treatment with intranasal TAA is associated with significant improvements in PAR-related QoL over 4 weeks, as measured using the miniRQLQ.

\section{Acknowledgments}

We thank the investigators, nurses, and participants who made this study possible and all members of the NCT03317015 clinical project team for their commitment to the study. Editorial support was provided by Kezia Pittaway, MSc, and Ella Palmer, PhD, CMPP, of inScience Communications, Springer Healthcare Ltd., UK, and was funded by Sanofi. The authors would like to thank Beatrice Bois De Fer and Aurore Allard for the critical reading of the manuscript.

\section{Statement of Ethics}

This study was conducted in accordance with the Declaration of Helsinki, the International Council for Harmonization (ICH) Good Clinical Practice (GCP) guidelines, and Russian regulations. Informed consent was obtained from all participants prior to their enrollment. The study protocol was approved by the research ethics committees at each study site.

\section{Conflict of Interest Statement}

A.V.K. acted as a clinical trial investigator during the study. N.N. has received consulting fees, honoraria for lectures, and/or research funding from AstraZeneca, ALK-Abello, Boehringer Ingelheim, Glenmark, MSD, Novartis, Sanofi, and Stallergenes Greer. Y.S. declares no conflicts of interest. A.M. and L.L. are Sanofi employees.

\section{Funding Sources}

This study was sponsored by Sanofi.

\section{Author Contributions}

A.V.K. supervised the design of the study and acted as a clinical investigator during the study. All authors made substantial contributions to the study conception and design and to data acquisition, analysis, and/or interpretation. All authors contributed to drafting the article or revising it critically for important intellectual content, and all have given their final approval of the version submitted for publication.

\section{Data Availability Statement}

The data that support the findings of this study are not publicly available due to Sanofi policies, but are available from Sanofi company upon reasonable request.

\section{References}

1 Bousquet J, Khaltaev N, Cruz AA, Denburg J, Fokkens WJ, Togias A, et al. Allergic rhinitis and its impact on asthma (ARIA) 2008 update (in collaboration with the World Health Organization, GA(2)LEN and AllerGen). Allergy. 2008 Apr;63 Suppl 86:8-160.

2 Khaitov RM, Ilyna NI. Allergology and immunology: national guidance. Moscow, Russia: GEOTAR-Media; 2014.

3 Bousquet PJ, Demoly P, Devillier P, Mesbah $\mathrm{K}$, Bousquet J. Impact of allergic rhinitis symptoms on quality of life in primary care. Int Arch Allergy Immunol. 2013;160(4):393400 .
4 Small P, Keith PK, Kim H. Allergic rhinitis. Allergy Asthma Clin Immunol. 2018;14(Suppl 2):51.

5 Maoua M, Maalel OE, Kacem I, Guedri S, Ben Kacem M, Aissa S, et al. Quality of life and work productivity impairment of patients with allergic occupational rhinitis. Tanaffos. 2019 Jan;18(1):58-65.

6 Greiner AN, Hellings PW, Rotiroti G, Scadding GK. Allergic rhinitis. Lancet. 2011 Dec 17;378(9809):2112-22

7 Elsammaa M. Registry on assessing the quality of life improvement with triamcinolone in the treatment of moderate-to-severe persis- tent allergic rhinitis in egyptian patients. Egyptian J Otolaryngol. 2016;32(4):243-7.

8 EMA. Nasacort Allergy 55 micrograms/dose Nasal Spray suspension; 2018.

9 Karaulov AV, Vylegzhanina T, Ovchinnikov A, Chernikova M, Nenasheva N. Triamcinolone acetonide versus fluticasone propionate in the treatment of perennial allergic rhinitis: a randomized, parallel-group trial. Int Arch Allergy Immunol. 2019;179(2):142-51.

10 Juniper EF, Thompson AK, Ferrie PJ, Roberts JN. Development and validation of the mini rhinoconjunctivitis quality of life questionnaire. Clin Exp Allergy. 2000 Jan;30(1):132-40. 
11 Gross G, Jacobs RL, Woodworth TH, Georges GC, Lim JC. Comparative efficacy, safety, and effect on quality of life of triamcinolone acetonide and fluticasone propionate aqueous nasal sprays in patients with fall seasonal allergic rhinitis. Ann Allergy Asthma Immunol. 2002 Jul;89(1):56-62.

12 Berger WE, Kaiser H, Gawchik SM, Tillinghast J, Woodworth TH, Dupclay L, et al. Triamcinolone acetonide aqueous nasal spray and fluticasone propionate are equally effective for relief of nasal symptoms in patients with seasonal allergic rhinitis. Otolaryngol Head Neck Surg. 2003 Jul;129(1):16-23.

13 Berger WE, Meltzer EO. Intranasal spray medications for maintenance therapy of allergic rhinitis. Am J Rhinol Allergy. 2015 JulAug;29(4):273-82.

14 Gawchik SM, Saccar CL. A risk-benefit assessment of intranasal triamcinolone acetonide in allergic rhinitis. Drug Saf. 2000 Oct; 23(4):309-22.
15 Schoenwetter W, Lim J. Comparison of intranasal triamcinolone acetonide with oral loratadine for the treatment of patients with seasonal allergic rhinitis. Clin Ther. 1995 May-Jun;17(3):479-92.

16 Condemi J, Schulz R, Lim J. Triamcinolone acetonide aqueous nasal spray versus loratadine in seasonal allergic rhinitis: efficacy and quality of life. Ann Allergy Asthma Immunol. 2000 May;84(5):533-8.

17 Bielory L, Gross GN, Letierce A, Melas-Melt L, Lucio L. Ocular symptoms improvement from intranasal triamcinolone compared with placebo and intranasal fluticasone propionate: a meta-analysis. Ann Allergy Asthma Immunol. 2020 Jun;124(6):616-21.e3.

18 Karaulov AV, Shartanova N, Maslakov A, Lucio L. Triamcinolone acetonide in the treatment of perennial allergic rhinitis: a post hoc efficacy analysis of a Phase III study performed in Russia. Int Arch Allergy Immunol. 2021. (submitted).

19 Mintz M, Garcia J, Diener P, Liao Y, Dupclay L, Georges G. Triamcinolone acetonide aqueous nasal spray improves nocturnal rhinitisrelated quality of life in patients treated in a primary care setting: the quality of sleep in Allergic Rhinitis study. Ann Allergy Asthma Immunol. 2004 Feb;92(2):255-61.
20 Herman H. Once-daily administration of intranasal corticosteroids for allergic rhinitis: a comparative review of efficacy, safety, patient preference, and cost. Am J Rhinol. 2007 JanFeb;21(1):70-9.

21 Sastre J, Mosges R. Local and systemic safety of intranasal corticosteroids. J Investig Allergol Clin Immunol. 2012;22(1):1-12.

22 Bridgeman MB. Overcoming barriers to intranasal corticosteroid use in patients with uncontrolled allergic rhinitis. Integr Pharm Res Pract. 2017;6:109-19.

23 Lipworth BJ, Jackson CM. Safety of inhaled and intranasal corticosteroids: lessons for the new millennium. Drug Saf. 2000 Jul;23(1): 11-33.

24 Ahmadiafshar A, Ahmadiafshar S. Efficacy and safety of inhaled and intranasal corticosteroids. Antiinflamm Antiallergy Agents Med Chem. 2014;13(2):83-7.

25 Stokes M, Amorosi SL, Thompson D, Dupclay L, Garcia J, Georges G. Evaluation of patients' preferences for triamcinolone acetonide aqueous, fluticasone propionate, and mometasone furoate nasal sprays in patients with allergic rhinitis. Otolaryngol Head Neck Surg. 2004 Sep;131(3):225-31. 